\title{
On the asymptotics of supremum distribution for some iterated processes
}

\author{
Marek Arendarczyk ${ }^{1}$ (1)
}

Received: 20 April 2016 / Revised: 19 September 2016 / Accepted: 20 September 2016/

Published online: 13 October 2016

(C) The Author(s) 2016. This article is published with open access at Springerlink.com

\begin{abstract}
In this paper, we study the asymptotic behavior of supremum distribution of some classes of iterated stochastic processes $\{X(Y(t)): t \in[0, \infty)\}$, where $\{X(t): t \in \mathbb{R}\}$ is a centered Gaussian process and $\{Y(t): t \in[0, \infty)\}$ is an independent of $\{X(t)\}$ stochastic process with a.s. continuous sample paths. In particular, the asymptotic behavior of $\mathbb{P}\left(\sup _{s \in[0, T]} X(Y(s))>u\right)$ as $u \rightarrow \infty$, where $T>0$, as well as $\lim _{u \rightarrow \infty} \mathbb{P}\left(\sup _{s \in[0, h(u)]} X(Y(s))>u\right)$, for some suitably chosen function $h(u)$ are analyzed. As an illustration, we study the asymptotic behavior of the supremum distribution of iterated fractional Brownian motion process.
\end{abstract}

Keywords Exact asymptotics · Supremum distribution · Iterated process · Iterated fractional brownian motion · Gaussian process

AMS 2000 Subject Classifications Primary 60G15 · 60G18 · Secondary 60G70

\section{Introduction}

Let $\{X(t): t \in \mathbb{R}\}$ and $\{Y(t): t \in[0, \infty)\}$ be two independent stochastic processes. This contribution is devoted to the analysis of asymptotic behavior of supremum distribution of iterated process $\{X(Y(t)): t \in[0, \infty)\}$.

Marek Arendarczyk

marendar@math.uni.wroc.pl

1 Mathematical Institute, University of Wrocław, pl. Grunwaldzki 2/4, 50-384, Wrocław, Poland 
Originated by Burdzy $(1993 ; 1994)$ for the case of iterated Brownian motion, the problem of analyzing the properties of iterated processes was intensively studied in recent years. Motivation for the analysis of the process $\{X(Y(t))\}$ in case of $\{X(t)\}$ and $\{Y(t)\}$ being independent Brownian motions was delivered by its connections to the 4th order PDE's (see, e.g., Funki 1979; Allouba and Zheng 2001; Nourdin and Peccati 2008). A vast literature is devoted to the analysis of many interesting probabilistic properties of iterated Brownian motions (see, e.g., Burdzy and Khoshnevisan 1995; Hu et al. 1995; Shi 1995; Bertoin 1996; Khoshnevisan and Lewis 1996; Eisenbaum and Shi 1999; Khoshnevisan and Lewis 1999). We also refer to (Curien and Konstantopoulos 2014) where convergence of finite dimensional distributions of $n$th iterated Brownian motion is studied and (Turban 2004) where infinite iterations of i.i.d. random walks are analyzed.

Recent studies also focus on properties of $\{X(Y(t)): t \in[0, \infty)\}$ for the case of more general Gaussian processes $\{X(t)\}$. One of interesting example of such processes is fractional Laplace motion $\left\{B_{H}(\Gamma(t)): t \in[0, \infty)\right\}$, where $\{\Gamma(t): t \in$ $[0, \infty)\}$ is a Gamma process. Motivation for analyzing fractional Laplace motions stems from hydrodynamic models (see, e.g., Kozubowski et al. 2004). This kind of processes were described in (Kozubowski et al. 2006), see also (Arendarczyk and Dȩbicki 2011) where asymptotic behavior of exit-time distribution for the process $\left\{B_{H}(\Gamma(t))\right\}$ was found. Another important class of iterated processes are the so-called $\alpha$-time fractional Brownian motions $\left\{B_{H}(Y(t))\right\}$, where $\{Y(t)\}$ is $\alpha$-stable subordinator independent of the process $\left\{B_{H}(t)\right\}$ (see, e.g., Linde and Shi 2004; Nane 2006; Linde and Zipfel 2008; Aurzada and Lifshits 2009). We also refer to (Michna 1998) and (Dȩbicki et al. 2014) where the process $\left\{B_{H}(Y(t))\right\}$ was analyzed in the context of theoretical actuarial models.

The process $\left\{B_{H}(Y(t))\right\}$ in the case of $\{Y(t)\}$ not being a subordinator was studied in (Aurzada and Lifshits 2009). In this case, the small deviations asymptotics was found for the so-called iterated fractional Brownian motion process $\left\{B_{H_{2}}\left(B_{H_{1}}(t)\right)\right\}$, where $\left\{B_{H_{1}}(t)\right\},\left\{B_{H_{2}}(t)\right\}$ are independent fractional Brownian motions with Hurst parameters $H_{1}, H_{2} \in(0,1]$ respectively.

In this paper, we focus on the analysis of asymptotic behavior of supremum distribution of the process $\{X(Y(t)): t \in[0, \infty)\}$ for general classes of stochastic processes $\{X(t)\},\{Y(t)\}$ with a.s. continuous sample paths.

Notation and organization of the paper:

In Section 2, we study the asymptotic behavior of

$$
\mathbb{P}\left(\sup _{s \in[0, T]} X(Y(s))>u\right) \text { as } u \rightarrow \infty,
$$


where $T>0$ and $\{X(t): t \in \mathbb{R}\},\{Y(t): t \in[0, \infty)\}$ are independent stochastic processes. This problem is closely related to the analysis of asymptotic behavior of the supremum distribution of the process $\{X(t)\}$ over a random time interval (see, e.g., Dȩbicki et al. 2004; Arendarczyk and Dȩbicki 2011; 2012; Tan and Hashorva 2013; Dębicki et al. 2014).

We start in Section 2.1 by giving general result for the case of $\{X(t)\}$ being Gaussian process with stationary increments and convex variance function (see Section 2.1, assumptions A1 - A3). In this case, under some general conditions on the process $\{Y(t)\}$ (see Section 2.1, assumptions L1, L2), we show that (1) reduces to

$$
\mathbb{P}\left(\sup _{s \in[0, \mathcal{T}]} X(s)>u\right) \text { as } u \rightarrow \infty
$$

where $\mathcal{T}$ is a non-negative random variable independent of $\{X(t)\}$ with asymptotically Weibullian tail distribution, that is,

$$
\mathbb{P}(\mathcal{T}>u)=C u^{\gamma} \exp \left(-\beta u^{\alpha}\right)(1+o(1))
$$

as $u \rightarrow \infty$, where $\alpha, \beta, C>0, \gamma \in \mathbb{R}$ (see, e.g., Arendarczyk and Dębicki (2011) for details). We write $\mathcal{T} \in \mathcal{W}(\alpha, \beta, \gamma, C)$ if $\mathcal{T}$ satisfies (3).

Section 2.2 is devoted to the special case of the process $\left\{B_{H}(Y(t)): t \in[0, \infty)\right\}$, where $\left\{B_{H}(t): t \in \mathbb{R}\right\}$ is a fractional Brownian motion $(f B m)$ with Hurst parameter $H \in(0,1]$, that is, a centered Gaussian process with stationary increments, a.s. continuous sample paths, $B_{H}(0)=0$, and covariance function $\operatorname{Cov}\left(B_{H}(t), B_{H}(s)\right)=$ $\frac{1}{2}\left(|s|^{2 H}+|t|^{2 H}-|t-s|^{2 H}\right)$. Due to self-similarity of the process $\left\{B_{H}(t)\right\}$, we are able to provide the exact asymptotics of (1) for the whole range of Hurst parameters $H \in(0,1]$. As an illustration, in Proposition 2.4, we work out the exact asymptotics of the supremum distribution of iterated fractional Brownian motion $\left\{B_{H_{2}}\left(B_{H_{1}}(t)\right): t \in[0, \infty)\right\}$, where $\left\{B_{H_{1}}(t)\right\},\left\{B_{H_{2}}(t)\right\}$ are independent fractional Brownian motions with Hurst parameters $H_{1}, H_{2}$ respectively. Note that small deviation counterpart of this problem was recently studied in (Aurzada and Lifshits 2009).

In Section 2.3, the case of $\{X(t)\}$ being a stationary Gaussian process is analyzed (see Section 2.3, assumptions D1, D2). In this case the exact asymptotics of (1) can be achieved under a general condition of finite average span of the process $\{Y(t)\}$ (see Section 2.3, assumption S1). This problem is strongly related to the analysis of (2) in case of $\mathcal{T}$ being a random variable with finite mean. In this case the asymptotics 
of (2) has the form (see Arendarczyk and Dębicki (2012), Theorem 3.1, and also Pickands (1969) for the classical result of Pickands' on deterministic time interval)

$$
\mathbb{P}\left(\sup _{s \in[0, \mathcal{T}]} X(s)>u\right)=\mathbb{E} \mathcal{T} C^{1 / \alpha} \mathcal{H}_{\alpha} u^{2 / \alpha} \Psi(u)(1+o(1))
$$

as $u \rightarrow \infty$, where $\mathcal{H}_{\alpha}$ is the Pickands' constant defined by the limit

$$
\mathcal{H}_{\alpha}=\lim _{T \rightarrow \infty} \frac{1}{T} \mathbb{E} \exp \left(\sup _{t \in[0, T]} \sqrt{2} B_{\frac{\alpha}{2}}(t)-t^{\alpha}\right)
$$

and $\Psi(u):=\mathbb{P}(\mathcal{N}>u)$ with $\mathcal{N}$ denoting the standard normal random variable.

In the second part of the paper, we study

$$
\lim _{u \rightarrow \infty} \mathbb{P}\left(\sup _{s \in[0, h(u)]} X(Y(s))>u\right)
$$

for some suitably chosen function $h(u)$.

First, in Theorem 3.1 we investigate limiting behavior of (4) for the case of $\{X(t)\}$ and $\{Y(t)\}$ being independent Gaussian processes with stationary increments that satisfy some general regularity conditions (see Section 3, assumptions B1 - B3). Then, in Theorem 3.2 and Proposition 3.3, the case of $\{X(t)\}$ being stationary Gaussian process is studied. We analyze $\{X(Y(t))\}$ for both weakly and strongly dependent stationary Gaussian processes $\{X(t)\}$ (see Section 3, assumptions D1 - D3). In these settings we provide (4) in the case of $\{Y(t)\}$ being a centered Gaussian process with stationary increments, as well as for self-similar process $\{Y(t)\}$ that is not necessarily Gaussian.

\section{Short timescale case}

In this section, we study the asymptotic behavior of

$$
\mathbb{P}\left(\sup _{s \in[0, T]} X(Y(s))>u\right) \text { as } u \rightarrow \infty
$$

where $T>0$, for the case of $\{X(t): t \in \mathbb{R}\}$ being a centered Gaussian process with a.s. continuous sample paths. We focus on two important classes of Gaussian processes. First, processes $\{X(t)\}$ with stationary increments are studied. Then, we analyze the case of stationary processes $\{X(t)\}$. 


\subsection{The stationary increments case}

Let $\{X(t): t \in \mathbb{R}\}$ be a centered Gaussian process with stationary increments, a.s. continuous sample paths, $X(0)=0$ a.s., and variance function $\sigma_{X}^{2}(t):=\operatorname{Var}(X(t))$ that satisfies the following assumptions

A1 $\sigma_{X}^{2}(t) \in C^{1}([0, \infty))$ is convex;

A2 $\sigma_{X}^{2}(t)$ is regularly varying at $\infty$ with parameter $\alpha_{\infty} \in(1,2)$;

A3 there exists $D>0$ such that $\sigma_{X}^{2}(t) \leq D t^{\alpha_{\infty}}$ for each $t \geq 0$.

To provide general result for (5) we assume that $\{Y(t): t \in[0, \infty)\}$ is a stochastic process with a.s. continuous sample paths, which is independent of $\{X(t)\}$ and its extremal distributions belong to the Weibullian class of random variables, that is,

L1 $\mathcal{M}:=\sup _{s \in[0, T]} Y(s) \in \mathcal{W}\left(\alpha_{1}, \beta_{1}, \gamma_{1}, C_{1}\right)$, with $\alpha_{1}, \beta_{1}, C_{1}>0, \gamma_{1} \in \mathbb{R}$

L2 $\mathcal{K}:=-\inf _{s \in[0, T]} Y(s) \in \mathcal{W}\left(\alpha_{2}, \beta_{2}, \gamma_{2}, C_{2}\right)$, with $\alpha_{2}, \beta_{2}, C_{2}>0, \gamma_{2} \in \mathbb{R}$.

Remark 2.1 Note that assumptions L1, L2 cover, e.g., a class of general Gaussian processes.

In the following theorem we present structural form of the asymptotics. The explicit asymptotic expansion is presented in Corollary 2.2.

Theorem 2.1 Let $\{X(t): t \in \mathbb{R}\}$ be a centered Gaussian process with stationary increments and variance function $\sigma_{X}^{2}(t)$ that satisfies assumptions $\mathbf{A 1}-\boldsymbol{A} \mathbf{3}$ and $\{Y(t): t \in[0, \infty)\}$ be an independent of $\{X(t)\}$ stochastic process with a.s. continuous sample paths that satisfies L1, L2. If

(i) $\mathbb{P}(\mathcal{K}>u)=o(\mathbb{P}(\mathcal{M}>u))$ as $u \rightarrow \infty$, then

$$
\mathbb{P}\left(\sup _{s \in[0, T]} X(Y(s))>u\right)=\mathbb{P}(X(\mathcal{M})>u)(1+o(1)) \text { as } u \rightarrow \infty
$$

(ii) $\mathbb{P}(\mathcal{M}>u)=o(\mathbb{P}(\mathcal{K}>u))$ as $u \rightarrow \infty$, then

$$
\mathbb{P}\left(\sup _{s \in[0, T]} X(Y(s))>u\right)=\mathbb{P}(X(\mathcal{K})>u)(1+o(1)) \text { as } u \rightarrow \infty
$$


(iii) $\mathbb{P}(\mathcal{K}>u)=\frac{C_{2}}{C_{1}} \mathbb{P}(\mathcal{M}>u)(1+o(1))$, as $u \rightarrow \infty$, then

$\mathbb{P}\left(\sup _{s \in[0, T]} X(Y(s))>u\right)=(\mathbb{P}(X(\mathcal{M})>u)+\mathbb{P}(X(\mathcal{K})>u))(1+o(1))$ as $u \rightarrow \infty$.

The proof of Theorem 2.1 is presented in Section 4.1.

If the variance function of $\{X(t)\}$ is regular enough, then the straightforward application of Corollary 3.2 in (Arendarczyk and Dȩbicki 2011) enables us to give the exact form of the asymptotics.

Corollary 2.2 Let $\{X(t): t \in \mathbb{R}\}$ be a centered Gaussian process with stationary increments and variance function that satisfies $\boldsymbol{A} \mathbf{1}$ and $\{Y(t): t \in[0, \infty)\}$ be an independent of $\{X(t)\}$ stochastic process with a.s. continuous sample paths that satisfies L1, L2. Additionally, if $\sigma_{X}^{2}(t)=D t^{\alpha_{\infty}}+o\left(t^{\alpha_{\infty}-\alpha}\right)$, as $t \rightarrow \infty$, with $\alpha_{\infty} \in(1,2)$, $D>0$, and $\alpha=\min \left(\alpha_{1}, \alpha_{2}\right)$, then

$$
\sup _{s \in[0, T]} X(Y(s)) \in \mathcal{W}(\widetilde{\alpha}, \widetilde{\beta}, \tilde{\gamma}, \widetilde{C}),
$$

where

$$
\begin{array}{ll}
\tilde{\alpha}=\frac{2 \alpha}{\alpha+\alpha_{\infty}}, \quad \tilde{\beta}=\beta^{\frac{\alpha \infty}{\alpha+\alpha_{\infty}}}\left(\frac{D}{2}\right)^{\frac{\alpha}{\alpha+\alpha_{\infty}}}\left(\left(\frac{\alpha}{\alpha_{\infty}}\right)^{\frac{\alpha_{\infty}}{\alpha+\alpha_{\infty}}}+\left(\frac{\alpha_{\infty}}{\alpha}\right)^{\frac{\alpha}{\alpha+\alpha_{\infty}}}\right), \\
\tilde{\gamma}=\frac{2 \gamma}{\alpha+\alpha_{\infty}}, \quad \tilde{C}=C D^{-1 / \alpha_{\infty}} \sqrt{\frac{\alpha_{\infty}}{2\left(\alpha+\alpha_{\infty}\right)}}\left(\frac{\alpha_{\infty}}{2 \alpha \beta} D^{\alpha_{\infty} / \alpha}\right)^{\frac{\gamma}{\alpha+\alpha_{\infty}}},
\end{array}
$$

with

$(\beta, \gamma, C)= \begin{cases}\left(\beta_{1}, \gamma_{1}, C_{1}\right) & \text { for } \mathbb{P}(\mathcal{K}>u)=o(\mathbb{P}(\mathcal{M}>u)) \text { as } u \rightarrow \infty \\ \left(\beta_{2}, \gamma_{2}, C_{2}\right) & \text { for } \mathbb{P}(\mathcal{M}>u)=o(\mathbb{P}(\mathcal{K}>u)) \text { as } u \rightarrow \infty \\ \left(\beta_{1}, \gamma_{1}, C_{1}+C_{2}\right) & \text { for } \mathbb{P}(\mathcal{K}>u)=\frac{C_{2}}{C_{1}} \mathbb{P}(\mathcal{M}>u)(1+o(1)) \text { as } u \rightarrow \infty\end{cases}$

\subsection{The case of $\mathrm{fBm}$}

Let $\left\{B_{H}(t): t \in \mathbb{R}\right\}$ be a fractional Brownian motion with Hurst parameter $H \in$ $(0,1]$. In this section, we analyze the asymptotic behavior of

$$
\mathbb{P}\left(\sup _{s \in[0, T]} B_{H}(Y(s))>u\right) \text { as } u \rightarrow \infty,
$$

where $T>0$ and $\{Y(t): t \in[0, \infty)\}$ is an independent of $\left\{B_{H}(t)\right\}$ stochastic process with a.s. continuous sample paths that satisfies assumptions L1, L2. Due to self-similarity of the process $\left\{B_{H}(t)\right\}$, we are able to provide the exact asymptotics 
of (6) for the whole range of Hurst parameters $H \in(0,1]$, which includes cases of both convex and concave variance functions.

Proposition 2.3 Let $\left\{B_{H}(t): t \in \mathbb{R}\right\}$ be a fractional Brownian motion with Hurst parameter $H \in(0,1]$ and $\{Y(t): t \in[0, \infty)\}$ be an independent of $\left\{B_{H}(t)\right\}$ stochastic process with a.s. continuous sample paths that satisfies L1, L2. If:

$H \in(0,1 / 2)$, then

$$
\sup _{s \in[0, T]} B_{H}(Y(s)) \in \mathcal{W}\left(\frac{2 \alpha}{\alpha+2 H}, \tilde{\beta}, \frac{2 \alpha-3 \alpha H+2 \gamma}{\alpha+2 H}, \tilde{C}_{1}\right),
$$

$H=1 / 2$, then

$$
\sup _{s \in[0, T]} B_{H}(Y(s)) \in \mathcal{W}\left(\frac{2 \alpha}{\alpha+2 H}, \tilde{\beta}, \frac{2 \gamma}{\alpha+2 H}, 2 \tilde{C}_{2}\right),
$$

$H \in(1 / 2,1]$, then

$$
\sup _{s \in[0, T]} B_{H}(Y(s)) \in \mathcal{W}\left(\frac{2 \alpha}{\alpha+2 H}, \tilde{\beta}, \frac{2 \gamma}{\alpha+2 H}, \tilde{C}_{2}\right),
$$

where

$$
\begin{aligned}
& \alpha=\min \left(\alpha_{1}, \alpha_{2}\right), \quad \tilde{\beta}=\beta^{\frac{2 H}{\alpha+2 H}}\left(\frac{1}{2}\left(\frac{\alpha}{H}\right)^{\frac{2 H}{\alpha+2 H}}+\left(\frac{H}{\alpha}\right)^{\frac{\alpha}{\alpha+2 H}}\right) \\
& \tilde{C}_{1}=\mathcal{H}_{H}\left(\frac{1}{2}\right)^{\frac{1}{2 H}} \frac{C}{\sqrt{\alpha+2 H}} H^{\frac{\alpha+6 H+2 \gamma-2}{2 \alpha+4 H}}(\alpha \beta)^{\frac{1-2 H-\gamma}{\alpha+2 H}}, \tilde{C}_{2}=\frac{C \sqrt{H}}{\sqrt{\alpha+2 H}}\left(\frac{H}{\alpha \beta}\right)^{\frac{\gamma}{\alpha+2 H}}
\end{aligned}
$$

with $\beta, \gamma, C$ as in Corollary 2.2.

The proof of Proposition 2.3 is presented in Section 4.2.

We now apply Proposition 2.3 to calculate the exact asymptotics for the special case of iterated fractional Brownian motion process $\left\{B_{H_{2}}\left(B_{H_{1}}(t)\right)\right\}$.

Proposition 2.4 Let $\left\{B_{H_{1}}(t): t \in \mathbb{R}\right\}$ and $\left\{B_{H_{2}}(t): t \in[0, \infty)\right\}$ be independent fractional Brownian motions with Hurst parameters $H_{1}, H_{2} \in[0,1)$ respectively. Then

$$
\sup _{s \in[0, T]} B_{H_{2}}\left(B_{H_{1}}(s)\right) \in \mathcal{W}(\alpha, \beta, \gamma, C),
$$

where

$$
\alpha=\frac{2}{H_{2}+1}, \quad \beta=\left(\frac{1}{T}\right)^{\frac{2 H_{1} H_{2}}{1+H_{2}}}\left(\frac{1}{2}\right)^{\frac{H_{2}}{1+H_{2}}}\left(\frac{1}{2}\left(\frac{2}{H_{2}}\right)^{\frac{H_{2}}{1+H_{2}}}+\left(\frac{H_{2}}{2}\right)^{\frac{1}{1+H_{2}}}\right),
$$


and

$$
(\gamma, C)=\left\{\begin{array}{l}
\left(\gamma_{1}, C_{1}\right) \text { for } H_{1} \in(0,1 / 2), H_{2} \in(0,1 / 2), \\
\left(\gamma_{2}, 2 C_{2}\right) \text { for } H_{1} \in(0,1 / 2), H_{2}=1 / 2, \\
\left(\gamma_{2}, C_{2}\right) \text { for } H_{1} \in(0,1 / 2), H_{2} \in(1 / 2,1] \\
\left(\gamma_{3}, 2 C_{3}\right) \text { for } H_{1}=1 / 2, H_{2} \in(0,1 / 2), \\
\left(\gamma_{4}, 4 C_{4}\right) \text { for } H_{1}=1 / 2, H_{2}=1 / 2, \\
\left(\gamma_{4}, 2 C_{4}\right) \text { for } H_{1}=1 / 2, H_{2} \in(1 / 2,1] \\
\left(\gamma_{3}, C_{3}\right) \text { for } H_{1} \in(1 / 2,1], H_{2} \in(0,1 / 2), \\
\left(\gamma_{4}, 2 C_{4}\right) \text { for } H_{1} \in(1 / 2,1], H_{2}=1 / 2, \\
\left(\gamma_{4}, C_{4}\right) \text { for } H_{1} \in(1 / 2,1], H_{2} \in(1 / 2,1],
\end{array}\right.
$$

with

$\gamma_{1}=\frac{1-H_{1}-3 H_{1} H_{2}}{H_{1}\left(1+H_{2}\right)}, \quad \gamma_{2}=\frac{1-3 H_{1}}{H_{1}\left(1+H_{2}\right)}, \quad \gamma_{3}=\frac{1-3 H_{2}}{1+H_{2}}, \quad \gamma_{4}=-\frac{1}{1+H_{2}}$,

$C_{1}=\left(\frac{1}{T}\right)^{H_{2}-3 H_{1} H_{2}} \frac{\mathcal{H}_{H_{1}} \mathcal{H}_{H_{2}}}{H_{1} \sqrt{\pi\left(1+H_{2}\right)}}\left(\frac{1}{2}\right)^{\frac{H_{1}+H_{2}+2 H_{1} H_{2}}{2 H_{1} H_{2}}} H_{2}^{\frac{1-3 H_{1}+3 H_{1} H_{2}}{2 H_{1}\left(1+H_{2}\right)}}$,

$C_{2}=\left(\frac{1}{T}\right)^{H_{2}-3 H_{1} H_{2}} \frac{\mathcal{H}_{H_{1}}}{H_{1} \sqrt{\pi\left(1+H_{2}\right)}}\left(\frac{1}{2}\right)^{\frac{1}{2 H_{1}}+1} H_{2}^{\frac{1-2 H_{1}+H_{1} H_{2}}{2 H_{1}\left(1+H_{2}\right)}}$,

$C_{3}=T^{H_{1} H_{2}} \frac{\mathcal{H}_{H_{2}}}{\sqrt{\pi\left(1+H_{2}\right)}}\left(\frac{1}{2}\right)^{\frac{1}{2 H_{2}}-1} H_{2}^{\frac{3 H_{2}-1}{2+2 H_{2}}}$,

$C_{4}=T^{H_{1} H_{2}} \frac{1}{2 \sqrt{\pi\left(1+H_{2}\right)}} H_{2}^{\frac{H_{2}}{2\left(1+H_{2}\right)}}$.

Proof Due to self-similarity of fBm

$$
\mathbb{P}\left(\sup _{s \in[0, T]} B_{H_{2}}\left(B_{H_{1}}(s)\right)>u\right)=\mathbb{P}\left(\sup _{s \in[0,1]} B_{H_{2}}\left(B_{H_{1}}(s)\right)>\frac{u}{T^{H_{1} H_{2}}}\right) .
$$

Moreover, due to Lemma 4.2 in (Arendarczyk and Dębicki 2011) (see also Piterbarg (1996), Theorem D3)

$$
\begin{gathered}
\sup _{s \in[0,1]} B_{H_{1}}(s) \in \mathcal{W}\left(2, \frac{1}{2}, \frac{1}{H_{1}}-3, \frac{\mathcal{H}_{H_{1}}}{H_{1} \sqrt{\pi}} 2^{-\frac{H_{1}+1}{2 H_{1}}}\right) \text { for } H_{1} \in(0,1 / 2) ; \\
\quad \sup _{s \in[0,1]} B_{H_{1}}(s) \in \mathcal{W}\left(2, \frac{1}{2},-1, \frac{2}{\sqrt{2 \pi}}\right) \text { for } H_{1}=1 / 2 ; \\
\sup _{s \in[0,1]} B_{H_{1}}(s) \in \mathcal{W}\left(2, \frac{1}{2},-1, \frac{1}{\sqrt{2 \pi}}\right) \text { for } H_{1} \in(1 / 2,1] .
\end{gathered}
$$


Additionally, by stationarity of the increments of $\mathrm{fBm}$

$$
-\inf _{s \in[0,1]} B_{H_{1}}(s) \stackrel{d}{=} \sup _{s \in[0,1]} B_{H_{1}}(s) .
$$

Now, in order to complete the proof it suffices to apply Proposition 2.3.

\subsection{The stationary case}

In this section, we analyze the asymptotic behavior of (5) for the case of $\{X(t): t \in$ $[0, \infty)\}$ being a centered stationary Gaussian process with a.s. continuous sample paths and covariance function $r(t):=\mathbb{C o v}(X(s), X(s+t))$. We impose the following assumptions on $r(t)$ (see, e.g., Piterbarg 1996):

D1 $\quad r(t)=1-C|t|^{\alpha}+o\left(|t|^{\alpha}\right)$ as $t \rightarrow 0$, with $\alpha \in(0,2]$ and $C>0$;

D2 $r(t)<1$ for all $t>0$.

In this case, we are able to give the exact form of the asymptotics for general class of stochastic processes $\{Y(t): t \in[0, \infty)\}$ that are independent of $\{X(t)\}$, have a.s. continuous sample paths and finite average span over interval $[0, T]$. Therefore, we assume that

S1 $\mathbb{E}\left[\sup _{s \in[0, T]} Y(s)-\inf _{s \in[0, T]} Y(s)\right]<\infty$.

Proposition 2.5 Let $\{X(t): t \in \mathbb{R}\}$ be a centered stationary Gaussian process with covariance function $r(t)$ that satisfies D1, D2 and $\{Y(t): t \in[0, \infty)\}$ be an independent of $\{X(t)\}$ stochastic process with a.s. continuous sample paths that satisfies S1. Then

$$
\mathbb{P}\left(\sup _{s \in[0, T]} X(Y(s))>u\right)=\mathbb{E}(\mathcal{T}) C^{\frac{1}{\alpha}} \mathcal{H}_{\alpha} u^{\frac{2}{\alpha}} \Psi(u)(1+o(1))
$$

as $u \rightarrow \infty$, where $\mathcal{T}=\sup _{s \in[0, T]} Y(s)-\inf _{s \in[0, T]} Y(s)$.

Proof Due to stationarity of the process $\{X(t)\}$, we have

$$
\begin{aligned}
\mathbb{P}\left(\sup _{t \in[0, T]} X(Y(t))>u\right) & =\mathbb{P}\left(\sup _{t \in\left[\inf _{s \in[0, T]} Y(s), \sup _{s \in[0, T]} Y(s)\right]} X(t)>u\right) \\
& =\mathbb{P}\left(\sup _{t \in[0, \mathcal{T}]} X(t)>u\right) .
\end{aligned}
$$

Now, in order to complete the proof it suffices to apply Theorem 3.1 in (Arendarczyk and Dȩbicki 2012). 
Remark 2.2 Equivalently, Proposition 2.5 states that

$$
\mathbb{P}\left(\sup _{t \in[0, T]} X(Y(t))>u\right)=\mathbb{E}(\mathcal{T}) \mathbb{P}\left(\sup _{t \in[0,1]} X(t)>u\right)(1+o(1))
$$

as $u \rightarrow \infty$, where $\mathcal{T}=\sup _{s \in[0, T]} Y(s)-\inf _{s \in[0, T]} Y(s)$.

\section{Long timescale case}

In this section, we investigate

$$
\lim _{u \rightarrow \infty} \mathbb{P}\left(\sup _{s \in[0, h(u)]} X(Y(s))>u\right)
$$

for a suitably chosen function $h(u)$.

In order to formulate the results, it is convenient to introduce the notation

$$
\sigma^{-1}(t):=\inf \{y \in[0, \infty): \sigma(y)>t\}
$$

for the generalized inverse of the function $\sigma(t)$.

We start with the observation that (7) can be straightforwardly obtained for any independent, self-similar processes $\{X(t)\}$ and $\{Y(t)\}$ with a.s. continuous sample paths.

Remark 3.1 Let $\{X(t): t \in \mathbb{R}\}$ and $\{Y(t): t \in[0, \infty)\}$ be independent, self-similar stochastic processes with a.s. continuous sample paths and self-similarity indexes $\lambda_{X}$ and $\lambda_{Y}$ respectively. Then, for $h(u)=u^{1 / \lambda_{X} \lambda_{Y}}(1+o(1))$ as $u \rightarrow \infty$, we have

$$
\lim _{u \rightarrow \infty} \mathbb{P}\left(\sup _{t \in[0, h(u)]} X(Y(t))>u\right)=\mathbb{P}\left(\sup _{t \in\left[\inf _{s \in[0,1]} Y(s), \sup _{s \in[0,1]} Y(s)\right]} X(t)>1\right) .
$$

In the next theorem, we extend this observation to the case of $\{X(t): t \in \mathbb{R}\}$ and $\{Y(t): t \in[0, \infty)\}$ being two independent, centered Gaussian processes with stationary increments, a.s. continuous sample paths, $X(0)=0$ and $Y(0)=0$ a.s., and variance functions $\sigma_{X}^{2}(t):=\mathbb{V} \operatorname{ar}(X(t))$ and $\sigma_{Y}^{2}(t):=\mathbb{V} \operatorname{ar}(Y(t))$ respectively. We assume that variance functions of both processes satisfy the following assumptions

B1 $\sigma^{2}(t) \in C([0, \infty))$ is ultimately strictly increasing ;

B2 $\sigma^{2}(t)$ is regularly varying at $\infty$ with parameter $\alpha \in(0,2]$;

B3 $\sigma^{2}(t)$ is regularly varying at 0 with parameter $\beta \in(0,2]$. 
In order to formulate the result, it is convenient to introduce the notation

$$
\mathcal{L}\left(\alpha_{X}, \alpha_{Y}\right)=\mathbb{P}\left(\sup _{t \in\left[\inf _{s \in[0,1]} B_{\alpha_{Y} / 2}(s), \sup _{s \in[0,1]} B_{\alpha_{Y} / 2}(s)\right]} B_{\alpha_{X} / 2}(t)>1\right),
$$

where $\left\{B_{\alpha_{X} / 2}(t)\right\},\left\{B_{\alpha_{Y} / 2}(t)\right\}$ are independent fractional Brownian motions with Hurst parameters $\alpha_{X} / 2$ and $\alpha_{Y} / 2$ respectively.

Theorem 3.1 Let $\{X(t): t \in \mathbb{R}\}$ and $\{Y(t): t \in[0, \infty)\}$ be independent, centered Gaussian processes with stationary increments that satisfy $\boldsymbol{B} \mathbf{1}-\boldsymbol{B} 3$ with parameters $\alpha_{X}, \beta_{X}, \alpha_{Y}, \beta_{Y}$ respectively. Then, for $h(u)=\sigma_{Y}^{-1}\left(\sigma_{X}^{-1}(u)\right)(1+o(1))$ as $u \rightarrow \infty$, we have

$$
\lim _{u \rightarrow \infty} \mathbb{P}\left(\sup _{t \in[0, h(u)]} X(Y(t))>u\right)=\mathcal{L}\left(\alpha_{X}, \alpha_{Y}\right)
$$

The proof of Theorem 3.1 is presented in Section 4.3.

The second part of this section focuses on the analysis of limiting behavior of (7) in the case of $\{X(t): t \in \mathbb{R}\}$ being a centered stationary Gaussian process with a.s. continuous sample paths and covariance function $r(t):=\operatorname{Cov}(X(s), X(s+t))$ that satisfies

D1 $\quad r(t)=1-C|t|^{\alpha}+o\left(|t|^{\alpha}\right)$ as $t \rightarrow 0$, with $\alpha \in(0,2]$ and $C>0$;

D2 $r(t)<1$ for all $t>0$;

D3 $r(t) \log (t) \rightarrow r$ as $t \rightarrow \infty$, with $r \in[0, \infty)$.

We study (7) for both weakly and strongly dependent stationary Gaussian processes, i.e., for $r=0$ and $r>0$ respectively. We refer to (Tan et al. 2012; Tan and Hashorva 2013) for recent results on asymptotic behavior of supremas of strongly dependent Gaussian processes.

In this settings, in Theorem 3.2, we provide (7) in the case of $\{Y(t): t \in[0, \infty)\}$ being a centered Gaussian process with stationary increments and variance function $\sigma_{Y}^{2}(t)$ that satisfies conditions B1 - B3. Moreover, in Proposition 3.3, we analyze (7) for self-similar process $\{Y(t)\}$ that is not necessarily Gaussian.

Theorem 3.2 Let $\{X(t): t \in \mathbb{R}\}$ be a centered stationary Gaussian process with covariance function that satisfies D1 - D3 and $\{Y(t): t \in[0, \infty)\}$ be an independent 
of $\{X(t)\}$ Gaussian process with a.s. continuous sample paths, stationary increments, and variance function $\sigma_{Y}^{2}(t)$ that satisfies $\boldsymbol{B} \mathbf{1}-\mathbf{B} 3$ with parameters $\alpha_{Y}, \beta_{Y}$. Then, for $h(u)=\sigma_{Y}^{-1}\left(\left(C^{\frac{1}{\alpha}} \mathcal{H}_{\alpha} u^{\frac{2}{\alpha}} \Psi(u)\right)^{-1}\right)(1+o(1))$ as $u \rightarrow \infty$,

we have

$$
\lim _{u \rightarrow \infty} \mathbb{P}\left(\sup _{s \in[0, h(u)]} X(Y(s))>u\right)=1-\mathbb{E} \exp (-\mathcal{T} \exp (-r+\sqrt{2 r} \mathcal{N})),
$$

where $\mathcal{T}=\sup _{s \in[0,1]} B_{\alpha_{Y} / 2}(s)-\inf _{s \in[0,1]} B_{\alpha_{Y} / 2}(s)$ and $\mathcal{N}$ is a normal random variable independent of $\mathcal{T}$.

The proof of Theorem 3.2 is given in Section 4.4 .

Proposition 3.3 Let $\{X(t): t \in \mathbb{R}\}$ be a stationary Gaussian process with covariance function that satisfies D1 - D3 and $\{Y(t): t \in[0, \infty)\}$ be a self-similar stochastic process with parameter $\lambda_{Y}$, independent of the process $\{X(t)\}$. Then, for $h(u)=\left[C^{\frac{1}{\alpha}} \mathcal{H}_{\alpha} u^{\frac{2}{\alpha}} \Psi(u)\right]^{-1 / \lambda_{Y}}(1+o(1))$ as $u \rightarrow \infty$, we have

$$
\lim _{u \rightarrow \infty} \mathbb{P}\left(\sup _{s \in[0, h(u)]} X(Y(s))>u\right)=1-\mathbb{E} \exp (-\mathcal{T} \exp (-r+\sqrt{2 r} \mathcal{N})),
$$

where $\mathcal{T}=\sup _{s \in[0,1]} Y(s)-\inf _{s \in[0,1]} Y(s)$ and $\mathcal{N}$ is a normal random variable independent of $\mathcal{T}$.

Proof Due to stationarity of the process $\{X(t)\}$ and self-similarity of the process $\{Y(t)\}$, we have

$$
\begin{aligned}
\mathbb{P}\left(\sup _{t \in[0, h(u)]} X(Y(t))>u\right) & =\mathbb{P}\left(\sup _{t \in\left[0, \sup _{s \in[0, h(u)]} Y(s)-\inf _{s \in[0, h(u)]} Y(s)\right]} X(t)>u\right) \\
& =\mathbb{P}\left(\sup _{t \in\left[0,(h(u))^{\lambda} Y \mathcal{T}\right]} X(t)>u\right) \\
& =1-\mathbb{E} \exp (-\mathcal{T} \exp (-r+\sqrt{2 r} \mathcal{N})),
\end{aligned}
$$

where (8) follows by the reasoning as in the proof of Theorem 3.2.

Remark 3.2 Note, that in the case of weakly dependent stationary Gaussian process $\{X(t)\}$, that is, if $r=0$ in $\mathbf{D 3}$, we obtain the following result

$$
\lim _{u \rightarrow \infty} \mathbb{P}\left(\sup _{s \in[0, h(u)]} X(Y(s))>u\right)=1-\mathbb{E} e^{-\mathcal{T}},
$$


where in the setting of Theorem 3.2, $h(u)=\sigma_{Y}^{-1}\left(\left(C^{\frac{1}{\alpha}} \mathcal{H}_{\alpha} u^{\frac{2}{\alpha}} \Psi(u)\right)^{-1}\right)(1+o(1))$ as $u \rightarrow \infty$, and $\mathcal{T}=\sup _{s \in[0,1]} B_{\alpha_{Y} / 2}(s)-\inf _{s \in[0,1]} B_{\alpha_{Y} / 2}(s)$; and in the setting of Proposition 3.3, $h(u)=\left[C^{\frac{1}{\alpha}} \mathcal{H}_{\alpha} u^{\frac{2}{\alpha}} \Psi(u)\right]^{-1 / \lambda_{Y}}(1+o(1))$ as $u \rightarrow \infty$, and $\mathcal{T}=$ $\sup _{s \in[0,1]} Y(s)-\inf _{s \in[0,1]} Y(s)$.

\section{Proofs}

In this section, we present detailed proofs of Theorem 2.1, Proposition 2.3, Theorem 3.1 and Theorem 3.2.

\subsection{Proof of Theorem 2.1}

In view of inclusion - exclusion principle

$$
\mathbb{P}\left(\sup _{s \in[0, T]} X(Y(s))>u\right)=P_{1}(u)+P_{2}(u)-P_{3}(u),
$$

where

$$
\begin{aligned}
P_{1}(u)=\mathbb{P}\left(\sup _{s \in[-\mathcal{K}, 0]} X(s)>u\right), \quad P_{2}(u)=\mathbb{P}\left(\sup _{s \in[0, \mathcal{M}]} X(s)>u\right), \\
P_{3}(u)=\mathbb{P}\left(\sup _{s \in[-\mathcal{K}, 0]} X(s)>u, \sup _{s \in[0, \mathcal{M}]} X(s)>u\right) .
\end{aligned}
$$

Observe that by definition of the process $\{X(t)\}$,

$$
P_{1}(u)=\mathbb{P}\left(\sup _{s \in[0, \mathcal{K}]} X(s)>u\right) .
$$

The case (i) is a consequence of the fact that, by (10) and Theorem 3.1 in (Arendarczyk and Dȩbicki 2011), $\mathbb{P}(\mathcal{K}>u)=o(\mathbb{P}(\mathcal{M}>u))$ implies $P_{1}(u)=o\left(P_{2}(u)\right)$. Thus,

$$
P_{2}(u) \leq \mathbb{P}\left(\sup _{s \in[0, T]} X(Y(s))>u\right) \leq P_{1}(u)+P_{2}(u)=P_{2}(u)(1+o(1))
$$

as $u \rightarrow \infty$, which in view of Theorem 3.1 in (Arendarczyk and Dębicki 2011), completes the proof for the case (i). A similar reasoning implies that for the case (ii), we have

$$
P_{1}(u) \leq \mathbb{P}\left(\sup _{s \in[0, T]} X(Y(s))>u\right) \leq P_{1}(u)+P_{2}(u)=P_{1}(u)(1+o(1))
$$


as $u \rightarrow \infty$, which in view of Theorem 3.1 in (Arendarczyk and Dębicki 2011), completes the proof for the case (ii).

In order to prove (iii), without loss of generality, we assume that

$$
\mathbb{P}(\mathcal{M}>u) \geq \mathbb{P}(\mathcal{K}>u)(1+o(1))
$$

as $u \rightarrow \infty$. Due to (9) combined with (10) and Theorem 3.1 in (Arendarczyk and Dębicki 2011), it suffices to show that $P_{3}(u)$ is negligible. We distinguish the case $\mathcal{K} \leq \mathcal{M}$ and the case $\mathcal{K}>\mathcal{M}$ and obtain

$$
\begin{aligned}
P_{3}(u) & =\mathbb{P}\left(\sup _{s \in[-\mathcal{K}, 0]} X(s)>u, \sup _{s \in[0, \mathcal{M}]} X(s)>u, \mathcal{K} \leq \mathcal{M}\right) \\
& +\mathbb{P}\left(\sup _{s \in[-\mathcal{K}, 0]} X(s)>u, \sup _{s \in[0, \mathcal{M}]} X(s)>u, \mathcal{K}>\mathcal{M}\right) \\
& \leq \mathbb{P}\left(\sup _{s \in[-\mathcal{M}, 0]} X(s)>u, \sup _{s \in[0, \mathcal{M}]} X(s)>u\right) \\
& +\mathbb{P}\left(\sup _{s \in[-\mathcal{K}, 0]} X(s)>u, \sup _{s \in[0, \mathcal{K}]} X(s)>u\right) \\
& \leq 2 \mathbb{P}\left(\sup _{s \in[-\mathcal{M}, 0]} X(s)>u, \sup _{s \in[0, \mathcal{M}]} X(s)>u\right)(1+o(1))
\end{aligned}
$$

as $u \rightarrow \infty$, where (12) is due to the assumption (11).

To find an upper bound of (12) it is convenient to make the following decomposition

$$
\begin{aligned}
& \mathbb{P}\left(\sup _{s \in[-\mathcal{M}, 0]} X(s)>u, \sup _{s \in[0, \mathcal{M}]} X(s)>u\right) \\
& =\left(\int_{0}^{a(u)}+\int_{a(u)}^{A(u)}+\int_{A(u)}^{\infty}\right) \mathbb{P}\left(\sup _{s \in[-w, 0]} X(s)>u, \sup _{s \in[0, w]} X(s)>u\right) d F_{\mathcal{M}}(w) \\
& =I_{1}+I_{2}+I_{3},
\end{aligned}
$$

where

$$
a(u)=u^{\frac{2}{\alpha \infty+2 \alpha_{1}}}, A(u)=u^{\frac{4}{2 \alpha_{\infty}+\alpha_{1}}}
$$


Let $\varepsilon>0$. We analyze each of the integrals $I_{1}, I_{2}, I_{3}$ separately.

Integral $I_{1}$ :

$$
\begin{aligned}
I_{1} & \leq \int_{0}^{a(u)} \mathbb{P}\left(\sup _{s \in[0, w]} X(s)>u\right) d F_{\mathcal{M}}(w) \\
& \leq \mathbb{P}\left(\sup _{s \in[0, a(u)]} X(s)>u\right) \\
& \leq \operatorname{Const} a(u)\left(\frac{u}{\sigma_{X}(a(u))}\right)^{\frac{2}{\alpha_{\infty}}} \Psi\left(\frac{u}{\sigma_{X}(a(u))}\right) \\
& \leq \exp \left(-u^{\frac{2 \alpha_{1}}{\alpha_{1}+\alpha_{\infty}}+\varepsilon}\right)(1+o(1))
\end{aligned}
$$

as $u \rightarrow \infty$, where (14) is due to (16) in (Arendarczyk and Dębicki 2011) (see also the proof of Lemma 6.3 in Arendarczyk and Dçbicki 2011).

Integral $\mathrm{I}_{3}$ :

$$
\begin{aligned}
I_{3} & \leq \int_{A(u)}^{\infty} \mathbb{P}\left(\sup _{s \in[0, w]} X(s)>u\right) d F_{\mathcal{M}}(w) \\
& \leq \mathbb{P}(\mathcal{M}>A(u)) \\
& =C_{1}(A(u))^{\gamma_{1}} \exp \left(-\beta_{1}(A(u))^{\alpha_{1}}\right)(1+o(1)) \\
& \leq \exp \left(-u^{\frac{2 \alpha_{1}}{\alpha_{1}+\alpha_{\infty}}+\varepsilon}\right)(1+o(1))
\end{aligned}
$$

as $u \rightarrow \infty$.

The above, combined with the observation that for each $\eta>0$ and sufficiently large $u$,

$$
\begin{aligned}
\mathbb{P}(X(\mathcal{M})>u) & =\mathbb{P}\left(\sigma_{X}(\mathcal{M}) \mathcal{N}>u\right) \geq \mathbb{P}\left(\sigma_{X}(\mathcal{M})>u^{\frac{\alpha \infty}{\alpha_{1}+\alpha_{\infty}}}\right) \mathbb{P}\left(\mathcal{N}>u^{\frac{\alpha_{1}}{\alpha_{1}+\alpha_{\infty}}}\right) \\
& \geq \exp \left(-u^{\frac{2 \alpha_{1}}{\alpha_{1}+\alpha_{\infty}}+\eta}\right)
\end{aligned}
$$

leads to the conclusion that $I_{1}$ and $I_{3}$ are negligible.

Integral $I_{2}$ : Observe that, due to $\mathbf{A 1}, \sigma_{X}^{2}(|t|) \leq \sigma_{X}^{2}(|t-s|)$, for each $(s, t) \in[-w, 0] \times$ $[0, w]$. Hence

$$
\operatorname{Var}(X(s)+X(t))=2 \sigma_{X}^{2}(|s|)+2 \sigma_{X}^{2}(|t|)-\sigma_{X}^{2}(|t-s|) \leq 3 \sigma_{X}^{2}(w)
$$


for $(s, t) \in[-w, 0] \times[0, w]$. Thus, according to the Borell inequality (see, e.g., Adler (1990), Theorem 2.1), combined with (15), $I_{2}$ is bounded by

$$
\begin{aligned}
& \int_{a(u)}^{A(u)} \mathbb{P}\left(\sup _{(s, t) \in[-w, 0] \times[0, w]}[X(s)+X(t)]>2 u\right) d F_{\mathcal{M}}(w) \\
& \leq 2 \int_{a(u)}^{A(u)} \exp \left(-\frac{2 u^{2}}{3 \sigma_{X}^{2}(w)}\left(1-\frac{1}{2 u} \mathbb{E}\left(\sup _{(s, t) \in[-w, 0] \times[0, w]}[X(s)+X(t)]\right)\right)^{2}\right) d F_{\mathcal{M}}(w) .(16)
\end{aligned}
$$

Moreover,

$$
0 \leq \mathbb{E}\left(\sup _{(s, t) \in[-w, 0] \times[0, w]}[X(s)+X(t)]\right) \leq 2 \mathbb{E}\left(\sup _{s \in[0, w]} X(t)\right) .
$$

To find the upper bound of $\mathbb{E} \sup _{t \in[0, w]} X(t)$, we use metric entropy method (see, e.g., Lifshits (2012), Chapter 10). At the beginning, for any $\mathbb{T} \subseteq \mathbb{R}$ define the semimetric

$$
d(s, t):=\sqrt{\mathbb{E}|X(t)-X(s)|^{2}}=\sigma_{X}(|t-s|) .
$$

The metric entropy $\mathbb{H}_{d}(\mathbb{T}, \epsilon)$ is defined as $\log N_{d}(\mathbb{T}, \epsilon)$, where $N_{d}(\mathbb{T}, \epsilon)$ denotes the minimal number of points in an $\epsilon$-net in $\mathbb{T}$ with respect to the semimetric $d$.

Observe that for $\mathbb{T}=[0, w]$,

$$
N_{d}(\mathbb{T}, \epsilon) \leq \frac{2 w}{\sigma_{X}^{-1}(\epsilon)},
$$

which, in view of Theorem 10.1 in (Lifshits 2012), implies that

$$
\begin{aligned}
\mathbb{E} \sup _{t \in[0, w]} X(t) & \leq 4 \sqrt{2} \int_{0}^{\sigma_{X}(w)} \sqrt{\log \frac{2 w}{\sigma_{X}^{-1}(\epsilon)}} d \epsilon \\
& \leq 4 \sqrt{2} \int_{0}^{\sqrt{D} w^{\frac{\alpha \infty}{2}}} \sqrt{\log \frac{\frac{2 D^{\frac{1}{\alpha \infty}} w}{\epsilon^{\frac{2}{\alpha}}}}{2}} d \epsilon \\
& =4 \sqrt{2} \int_{1 / w}^{\infty} \frac{\sqrt{D} \alpha_{\infty}}{2} x^{-\frac{\alpha_{\infty}}{2}-1} \sqrt{\log 2 w x} d x \\
& \leq 4 \alpha_{\infty} \sqrt{D w} \int_{1 / w}^{\infty} x^{-\frac{\alpha_{\infty}+1}{2}} d x \\
& \leq B w^{\frac{\alpha \infty}{2}},
\end{aligned}
$$

where $B=\frac{8 \sqrt{D} \alpha_{\infty}}{\alpha_{\infty}-1},(18)$ is due to $\mathbf{A 3}$, and (19) is by substitution $x:=D^{1 / \alpha_{\infty}} \epsilon^{-2 / \alpha_{\infty}}$. Finally, due to (17) combined with (20) and (13)

$$
0 \leq \frac{1}{2 u} \mathbb{E}\left(\sup _{(s, t) \in[-w, 0] \times[0, w]}[X(s)+X(t)]\right) \leq B \frac{w^{\frac{\alpha \infty}{2}}}{u} \leq B u^{-\frac{\alpha_{1}}{2 \alpha_{\infty}+\alpha_{1}}}
$$


for each $w \in[a(u), A(u)]$, which implies that

$$
\left(1-\frac{1}{2 u} \mathbb{E}\left(\sup _{(s, t) \in[-w, 0] \times[0, w]}[X(s)+X(t)]\right)\right)^{2} \rightarrow 1
$$

as $u \rightarrow \infty$, uniformly for $w \in[a(u), A(u)]$, and hence

$$
\exp \left(-\frac{2 u^{2}}{3 \sigma_{X}^{2}(w)}\left(1-\frac{1}{2 u} \mathbb{E}\left(\sup _{(s, t) \in[-w, 0] \times[0, w]}[X(s)+X(t)]\right)\right)^{2}\right)=o\left(\Psi\left(\frac{u}{\sigma_{X}(w)}\right)\right)
$$

as $u \rightarrow \infty$, uniformly for $w \in[a(u), A(u)]$. Thus, combining (16) with (21), we obtain, for sufficiently large $u$, the following upper bound,

$$
I_{2} \leq 2 \varepsilon \int_{a(u)}^{A(u)} \Psi\left(\frac{u}{\sigma_{X}(w)}\right) d F_{\mathcal{M}}(w) \leq 2 \varepsilon \mathbb{P}\left(\sup _{s \in[0, \mathcal{M}]} X(s)>u\right),
$$

which in view of Theorem 3.1 in (Arendarczyk and Dȩbicki 2011), implies that

$$
\limsup _{u \rightarrow \infty} \frac{I_{2}}{\mathbb{P}(X(\mathcal{M})>u)} \leq 2 \varepsilon .
$$

In order to complete the proof it suffices to pass with $\varepsilon \rightarrow 0$.

\subsection{Proof of Proposition 2.3}

The idea of the proof is analogous to the proof of Theorem 2.1, thus we present only main steps of the argumentation. In view of inclusion - exclusion principle

$$
\mathbb{P}\left(\sup _{s \in[0, T]} B_{H}(Y(s))>u\right)=P_{1}(u)+P_{2}(u)-P_{3}(u),
$$

where

$$
P_{1}(u)=\mathbb{P}\left(\sup _{s \in[-\mathcal{K}, 0]} B_{H}(s)>u\right), \quad P_{2}(u)=\mathbb{P}\left(\sup _{s \in[0, \mathcal{M}]} B_{H}(s)>u\right)
$$

and

$$
P_{3}(u)=\mathbb{P}\left(\sup _{s \in[-\mathcal{K}, 0]} B_{H}(s)>u, \sup _{s \in[0, \mathcal{M}]} B_{H}(s)>u\right) .
$$

Moreover observe that

$$
P_{1}(u)=\mathbb{P}\left(\sup _{s \in[0, \mathcal{K}]} B_{H}(s)>u\right) .
$$

Since the arguments for the cases $\mathbb{P}(\mathcal{K}>u)=o(\mathbb{P}(\mathcal{M}>u))$ as $u \rightarrow \infty$, and $\mathbb{P}(\mathcal{M}>u)=o(\mathbb{P}(\mathcal{K}>u))$ as $u \rightarrow \infty$ are similar to those in the proof of Theorem 2.1 , then we focus on the case $\mathbb{P}(\mathcal{K}>u)=\frac{C_{2}}{C_{1}} \mathbb{P}(\mathcal{M}>u)(1+o(1))$ as $u \rightarrow \infty$. 
Without loss of generality, we assume that

$$
\mathbb{P}(\mathcal{M}>u) \geq \mathbb{P}(\mathcal{K}>u)(1+o(1))
$$

as $u \rightarrow \infty$. Due to (22) combined with (23) and Theorem 4.1 in (Arendarczyk and Dȩbicki 2011), it suffices to show that $P_{3}(u)$ is negligible. In an analogous way to (12), we obtain the following upper bound

$$
P_{3}(u) \leq 2 \mathbb{P}\left(\sup _{s \in[-\mathcal{M}, 0]} B_{H}(s)>u, \sup _{s \in[0, \mathcal{M}]} B_{H}(s)>u\right)(1+o(1))
$$

as $u \rightarrow \infty$. Then, we consider decomposition

$$
\begin{aligned}
& \mathbb{P}\left(\sup _{s \in[-\mathcal{M}, 0]} B_{H}(s)>u, \sup _{s \in[0, \mathcal{M}]} B_{H}(s)>u\right) \\
& =\left(\int_{0}^{a(u)}+\int_{a(u)}^{A(u)}+\int_{A(u)}^{\infty}\right) \mathbb{P}\left(\sup _{s \in[-w, 0]} B_{H}(s)>u, \sup _{s \in[0, w]} B_{H}(s)>u\right) d F_{\mathcal{M}}(w) \\
& =I_{1}+I_{2}+I_{3},
\end{aligned}
$$

where

$$
a(u)=u^{\frac{1}{H+\alpha_{1}}}, \quad A(u)=u^{\frac{4}{4 H+\alpha_{1}}} .
$$

Let $\varepsilon>0$. We investigate the asymptotic behavior of each of the integrals.

Integral $I_{1}$ : Due to self-similarity of $\left\{B_{H}(t)\right\}$ combined with Lemma 4.2 in (Arendarczyk and Dȩbicki 2011), we have, as $u \rightarrow \infty$,

$I_{1} \leq \mathbb{P}\left(\sup _{s \in[0, a(u)]} B_{H}(s)>u\right)=\mathbb{P}\left(\sup _{s \in[0,1]} B_{H}(s)>\frac{u}{(a(u))^{H}}\right) \leq \exp \left(-u^{\frac{2 \alpha_{1}}{\alpha_{1}+H}+\varepsilon}\right)(1+o(1))$.

Integral $I_{3}$ : We have, as $u \rightarrow \infty$,

$$
I_{3} \leq \mathbb{P}(\mathcal{M}>A(u)) \leq \exp \left(-u^{\frac{2 \alpha_{1}}{\alpha_{1}+H}+\varepsilon}\right)(1+o(1)) .
$$

Observe that, due to Theorem 4.1 in (Arendarczyk and Dȩbicki 2011), for each $\eta>0$ and sufficiently large $u$,

$$
\mathbb{P}\left(\sup _{s \in[0, \mathcal{M}]} B_{H}(s)>u\right) \geq \exp \left(-u^{\frac{2 \alpha_{1}}{\alpha_{1}+H}+\eta}\right)(1+o(1))
$$

as $u \rightarrow \infty$. Thus, we conclude that $I_{1}$ and $I_{3}$ are negligible.

Integral $I_{2}$ : Observe that $|t|^{2 H} \leq|t-s|^{2 H}$, for each $(s, t) \in[-w, 0] \times[0, w]$. Hence

$$
\operatorname{Var}\left(B_{H}(s)+B_{H}(t)\right)=2|s|^{2 H}+2|t|^{2 H}-|t-s|^{2 H} \leq 3 w^{2 H}
$$


for $(s, t) \in[-w, 0] \times[0, w]$. Thus, according to the Borell inequality (see, e.g., Adler (1990), Theorem 2.1), combined with (25), $I_{2}$ is bounded by

$$
\begin{aligned}
& \int_{a(u)}^{A(u)} \mathbb{P}\left(\sup _{(s, t) \in[-w, 0] \times[0, w]}\left[B_{H}(s)+B_{H}(t)\right]>2 u\right) d F_{\mathcal{M}}(w) \\
& \leq 2 \int_{a(u)}^{A(u)} \exp \left(-\frac{2 u^{2}}{3 w^{2 H}}\left(1-\frac{1}{2 u} \mathbb{E}\left(\sup _{(s, t) \in[-w, 0] \times[0, w]}\left[B_{H}(s)+B_{H}(t)\right]\right)\right)^{2}\right) d F_{\mathcal{M}}(w) .(26)
\end{aligned}
$$

Moreover, due to self-similarity of $\left\{B_{H}(t)\right\}$

$$
0 \leq \mathbb{E}\left(\sup _{(s, t) \in[-w, 0] \times[0, w]}\left[B_{H}(s)+B_{H}(t)\right]\right) \leq 2 \mathbb{E}\left(\sup _{s \in[0, w]} B_{H}(s)\right)=B w^{H},
$$

where $B=2 \mathbb{E} \sup _{s \in[0,1]} B_{H}(s)$, which due to (24), implies that

$$
\left(1-\frac{1}{2 u} \mathbb{E}\left(\sup _{(s, t) \in[-w, 0] \times[0, w]}\left[B_{H}(s)+B_{H}(t)\right]\right)\right)^{2} \rightarrow 1
$$

as $u \rightarrow \infty$, uniformly for $w \in[a(u), A(u)]$, and hence

$$
\exp \left(-\frac{2 u^{2}}{3 w^{2 H}}\left(1-\frac{1}{2 u} \mathbb{E}\left(\sup _{(s, t) \in[-w, 0] \times[0, w]}\left[B_{H}(s)+B_{H}(t)\right]\right)\right)^{2}\right)=o\left(\Psi\left(\frac{u}{w^{H}}\right)\right)
$$

as $u \rightarrow \infty$, uniformly for $w \in[a(u), A(u)]$. Thus, combining (26) with (27), we obtain, for sufficiently large $u$, the following upper bound,

$$
I_{2} \leq 2 \varepsilon \int_{a(u)}^{A(u)} \Psi\left(\frac{u}{\sigma_{X}(w)}\right) d F_{\mathcal{M}}(w) \leq 2 \varepsilon \mathbb{P}\left(\sup _{s \in[0, \mathcal{M}]} X(s)>u\right),
$$

which in view of Theorem 4.1 in (Arendarczyk and Dȩbicki 2011), implies that

$$
\limsup _{u \rightarrow \infty} \frac{I_{2}}{\mathbb{P}(X(\mathcal{M})>u)} \leq 2 \varepsilon
$$

In order to complete the proof it suffices to pass with $\varepsilon \rightarrow 0$.

\subsection{Proof of Theorem 3.1}

In further analysis we use the following notation

$$
X_{\sigma_{Y}(h(u))}(s):=\frac{X\left(\sigma_{Y}(h(u)) s\right)}{\sigma_{X}\left(\sigma_{Y}(h(u))\right)} \quad \text { and } \quad Y_{h(u)}(s):=\frac{Y(h(u) s)}{\sigma_{Y}(h(u))} .
$$

Moreover, we denote

$$
\begin{aligned}
\mathcal{V}_{u}:=\inf _{s \in[0,1]} Y_{h(u)}(s), \quad \mathcal{W}_{u}:=\sup _{s \in[0,1]} Y_{h(u)}(s), \\
\mathcal{V}:=\inf _{s \in[0,1]} B_{\alpha_{Y} / 2}(s) \quad \mathcal{W}:=\sup _{s \in[0,1]} B_{\alpha_{Y} / 2}(s)
\end{aligned}
$$


Let $\varepsilon>0$ and $0<A_{\infty}<\infty$. We start with the observation that $\lim _{u \rightarrow \infty} h(u)=$ $\infty$, which also implies that $\lim _{u \rightarrow \infty} \sigma_{Y}(h(u))=\infty$. Hence, due to Lemma 5.2 in (Dȩbicki et al. 2004)

$$
\left(\mathcal{V}_{u}, \mathcal{W}_{u}\right) \Rightarrow(\mathcal{V}, \mathcal{W}) \text { as } u \rightarrow \infty
$$

and

$$
\sup _{s \in[v, w]} X_{\sigma_{Y}(h(u))}(s) \Rightarrow \sup _{s \in[v, w]} B_{\alpha_{X} / 2}(s) \text { as } u \rightarrow \infty
$$

uniformly for $(v, w) \in\left[-A_{\infty}, 0\right] \times\left[0, A_{\infty}\right]$, where $\Rightarrow$ denotes convergence in distribution.

By continuity of the sample paths of the processes $\{X(t)\}$ and $\{Y(t)\}$,

$$
\begin{aligned}
& \mathbb{P}\left(\sup _{t \in[0, h(u)]} X(Y(t))>u\right) \\
& =\mathbb{P}\left(\sup _{t \in\left[\inf _{s \in[0, h(u)]} Y(s), \sup _{s \in[0, h(u)]} Y(s)\right]} X(t)>u\right) \\
& =\mathbb{P}\left(\sup _{t \in\left[\mathcal{V}_{u}, \mathcal{W}_{u}\right]} X\left(\sigma_{Y}(h(u)) t\right)>u\right) \\
& =\mathbb{P}\left(\sup _{t \in\left[\mathcal{V}_{u}, \mathcal{W}_{u}\right]} X_{\sigma_{Y}(h(u))}(t)>\frac{u}{\sigma_{X}\left(\sigma_{Y}(h(u))\right)}\right) .
\end{aligned}
$$

To find an upper bound of (30) we consider the following decomposition

$$
\begin{aligned}
& \mathbb{P}\left(\sup _{t \in\left[\mathcal{V}_{u}, \mathcal{W}_{u}\right]} X_{\sigma_{Y}(h(u))}(t)>\frac{u}{\sigma_{X}\left(\sigma_{Y}(h(u))\right.}\right) \\
& \leq\left(\int_{-\infty}^{-A_{\infty}} \int_{0}^{\infty}+\int_{-A_{\infty}}^{0} \int_{0}^{A_{\infty}}+\int_{-\infty}^{0} \int_{A_{\infty}}^{\infty}\right) \mathbb{P}\left(\sup _{t \in[v, w]} X_{\sigma_{Y}(h(u))}(t)>\frac{u}{\sigma_{X}\left(\sigma_{Y}(h(u))\right)}\right) d_{\left(\mathcal{V}_{u}, \mathcal{W}_{u}\right)}(v, w) \\
& =I_{1}+I_{2}+I_{3} .
\end{aligned}
$$

We analyze each of the integrals $I_{1}, I_{2}, I_{3}$ separately.

Integral $I_{1}$ : Due to (28), for sufficiently large $u$,

$$
I_{1} \leq \mathbb{P}\left(\mathcal{V}_{u} \leq-A_{\infty}\right) \leq(1+\varepsilon) \mathbb{P}\left(\mathcal{V} \leq-A_{\infty}\right)
$$

Integral $I_{3}$ : Due to (28), for sufficiently large $u$,

$$
I_{3} \leq \mathbb{P}\left(\mathcal{W}_{u}>A_{\infty}\right) \leq(1+\varepsilon) \mathbb{P}\left(\mathcal{W}>A_{\infty}\right)
$$


Integral $I_{2}$ : For $u$ sufficiently large,

$$
\begin{aligned}
I_{2} & =\int_{-A_{\infty}}^{0} \int_{0}^{A_{\infty}} \mathbb{P}\left(\sup _{t \in[v, w]} X_{\sigma_{Y}(h(u))}(t)>\frac{u}{\sigma_{X}\left(\sigma_{Y}(h(u))\right)}\right) d_{\left(\mathcal{V}_{u}, \mathcal{W}_{u}\right)}(v, w) \\
& \leq(1+\varepsilon) \int_{-A_{\infty}}^{0} \int_{0}^{A_{\infty}} \mathbb{P}\left(\sup _{t \in[v, w]} B_{\alpha_{X} / 2}(t)>1\right) d_{\left(\mathcal{V}_{u}, \mathcal{W}_{u}\right)}(v, w) \\
& \leq(1+\varepsilon)^{2} \int_{-A_{\infty}}^{0} \int_{0}^{A_{\infty}} \mathbb{P}\left(\sup _{t \in[v, w]} B_{\alpha_{X} / 2}(t)>1\right) d_{(\mathcal{V}, \mathcal{W})}(v, w) \\
& \leq(1+\varepsilon)^{2} \mathbb{P}\left(\sup _{t \in[\mathcal{V}, \mathcal{W}]} B_{\alpha_{X} / 2}(t)>1\right),
\end{aligned}
$$

where (31) is due to (29) and the fact that $\lim _{u \rightarrow \infty} \frac{u}{\sigma_{X}\left(\sigma_{Y}(h(u))\right)}=1$, and (32) is due to (28), and the observation that $\mathbb{P}\left(\sup _{t \in[v, w]} B_{\alpha_{X} / 2}(t)>1\right)$ is bounded and continuous function with respect to $(v, w)$. Thus, for each $\varepsilon>0, A_{\infty}>0$,

$$
\begin{aligned}
\limsup _{u \rightarrow \infty} \mathbb{P}\left(\sup _{t \in[0, h(u)]} X(Y(t))>u\right) & \leq(1+\varepsilon)^{2} \mathbb{P}\left(\sup _{t \in[\mathcal{V}, \mathcal{W}]} B_{\alpha_{X} / 2}(t)>1\right) \\
& +(1+\varepsilon) \mathbb{P}\left(\mathcal{V} \leq-A_{\infty}\right)+(1+\varepsilon) \mathbb{P}\left(\mathcal{W}>A_{\infty}\right) .
\end{aligned}
$$

Analogously,

$$
\liminf _{u \rightarrow \infty} \mathbb{P}\left(\sup _{t \in[0, h(u)]} X(Y(t))>u\right) \geq(1-\varepsilon)^{2} \mathbb{P}\left(\sup _{t \in[\mathcal{V}, \mathcal{W}]} B_{\alpha_{X} / 2}(t)>1\right)
$$

In order to complete the proof it suffices to pass with $A_{\infty} \rightarrow \infty$, and $\varepsilon \rightarrow 0$.

\subsection{Proof of Theorem 3.2}

In further analysis we use the following notation

$$
\mathcal{T}_{u}:=\sup _{s \in[0,1]} Y_{h(u)}(s)-\inf _{s \in[0,1]} Y_{h(u)}(s), \quad \text { where } \quad Y_{h(u)}(s):=\frac{Y(h(u) s)}{\sigma_{Y}(h(u))}
$$

Let $\varepsilon>0$ and $0<A_{0}<A_{\infty}<\infty$. Note that due to Lemma 5.2 in (Dębicki et al. 2004)

$$
\mathcal{T}_{u} \Rightarrow \mathcal{T} \text { as } u \rightarrow \infty
$$


where $\Rightarrow$ denotes convergence in distribution.

It is convenient to consider the following decomposition

$$
\begin{aligned}
\mathbb{P}\left(\sup _{s \in[0, h(u)]} X(Y(s))>u\right) & =\mathbb{P}\left(\sup _{s \in\left[0, \mathcal{T}_{u} \sigma_{Y}(h(u))\right]} X(s)>u\right) \\
& =\left(\int_{0}^{A_{0}}+\int_{A_{0}}^{A_{\infty}}+\int_{A_{\infty}}^{\infty}\right) \mathbb{P}\left(\sup _{s \in\left[0, t \sigma_{Y}(h(u))\right]} X(s)>u\right) d F_{\mathcal{T}_{u}}(t) \\
& =I_{1}+I_{2}+I_{3} .
\end{aligned}
$$

We analyze each of the integrals $I_{1}, I_{2}, I_{3}$ separately.

Integral $I_{1}$ : Due to Lemma 3.3 in (Tan and Hashorva 2013), for sufficiently large $u$,

$$
\begin{aligned}
I_{1} & \leq \mathbb{P}\left(\sup _{s \in\left[0, A_{0} \sigma_{Y}(h(u))\right]} X(s)>u\right) \\
& \leq(1+\varepsilon)\left[1-\mathbb{E} \exp \left(-A_{0} \exp (-r+\sqrt{2 r} \mathcal{N})\right)\right]
\end{aligned}
$$

as $u \rightarrow \infty$.

Integral $I_{3}$ : Due to (33), for sufficiently large $u$,

$$
I_{3} \leq \mathbb{P}\left(\mathcal{T}_{u}>A_{\infty}\right) \leq(1+\varepsilon) \mathbb{P}\left(\mathcal{T}>A_{\infty}\right)
$$

Integral $I_{2}$ :

$$
\begin{aligned}
I_{2} & =\int_{A_{0}}^{A_{\infty}} \mathbb{P}\left(\sup _{s \in\left[0, t \sigma_{Y}(h(u))\right]} X(s)>u\right) d F_{\mathcal{T}_{u}}(t) \\
& \leq(1+\varepsilon) \int_{A_{0}}^{A_{\infty}}(1-\mathbb{E} \exp (-t \exp (-r+\sqrt{2 r} \mathcal{N}))) d F_{\mathcal{T}_{u}}(t) \\
& \leq(1+\varepsilon)^{2} \int_{A_{0}}^{A_{\infty}}(1-\mathbb{E} \exp (-t \exp (-r+\sqrt{2 r} \mathcal{N}))) d F_{\mathcal{T}}(t)
\end{aligned}
$$

where (34) is by Lemma 3.3 in (Tan and Hashorva 2013) and (35) is due to (33), and the observation that $1-\mathbb{E} \exp (-t \exp (-r+\sqrt{2 r} \mathcal{N}))$ is bounded and continuous function with respect to $t \in\left[A_{0}, A_{\infty}\right]$. Thus, for each $\varepsilon>0, A_{\infty}>A_{0}>0$,

$$
\begin{aligned}
\limsup _{u \rightarrow \infty} \mathbb{P}\left(\sup _{s \in[0, h(u)]} X(Y(s))>u\right) v & \leq(1+\varepsilon)^{2} \int_{A_{0}}^{A_{\infty}}(1-\mathbb{E} \exp (-t \exp (-r+\sqrt{2 r} \mathcal{N}))) d F_{\mathcal{T}}(t) \\
& +(1+\varepsilon)\left[1-\mathbb{E} \exp \left(-A_{0} \exp (-r+\sqrt{2 r} \mathcal{N})\right)\right] \\
& +(1+\varepsilon) \mathbb{P}\left(\mathcal{T}>A_{\infty}\right) .
\end{aligned}
$$


Analogously,

$\liminf _{u \rightarrow \infty} \mathbb{P}\left(\sup _{s \in[0, h(u)]} X(Y(s))>u\right) \geq(1-\varepsilon)^{2} \int_{A_{0}}^{A_{\infty}}(1-\mathbb{E} \exp (-t \exp (-r+\sqrt{2 r} \mathcal{N}))) d F_{\mathcal{T}}(t)$.

In order to complete the proof it suffices to pass with $A_{0} \rightarrow 0, A_{\infty} \rightarrow \infty$, and $\varepsilon \rightarrow 0$.

Acknowledgments I would like to thank Krzysztof Dębicki for a valuable review of an earlier version of the manuscript and anonymous referees for their useful comments and suggestions. This work was supported by NCN Grant No 2013/09/D/ST1/03698 (2014-2016).

Open Access This article is distributed under the terms of the Creative Commons Attribution 4.0 International License (http://creativecommons.org/licenses/by/4.0/), which permits unrestricted use, distribution, and reproduction in any medium, provided you give appropriate credit to the original author(s) and the source, provide a link to the Creative Commons license, and indicate if changes were made.

\section{References}

Adler, R.J.: An introduction to continuity, extrema, and related topics for general Gaussian processes Inst. Math. Statist. Lecture Notes -Monograph Series, vol. 12, Inst. Math. Statist., Hayward, CA (1990)

Allouba, H., Zheng, W.: Brownian-time processes: the PDE connection and the half-derivative generator. Ann. Probab. 29(4), 1780-1795 (2001)

Arendarczyk, M., Dębicki, K.: Asymptotics of supremum distribution of a Gaussian process over a Weibullian time. Bernoulli 17(1), 194-210 (2011)

Arendarczyk, M., Dębicki, K.: Exact asymptotics of supremum of a stationary Gaussian process over a random interval. Statist. Probab. Lett. 82, 645-652 (2012)

Aurzada, F., Lifshits, M.: On the small deviation problem for some iterated processes. Elec. J. Prob. 14(68), 1992-2010 (2009)

Bertoin, J.: Iterated Brownian motion and stable $\frac{1}{4}$ subordinator. Statist. Probab. Lett. 27(2), 111-114 (1996)

Burdzy, K.: Some path properties of iterated Brownian motion. Cinlar, E. (ed.) et al., Seminar on stochastic processes. Held at the Univ. of Washington, DC, USA, March 26-28, 1992. Basel: Birkhuser. Prog. Probab. 33, pp. 67-87 (1993)

Burdzy, K.: Variation of iterated Brownian motion. Dawson, D. A. (ed.), Measure-valued processes, stochastic partial differential equations, and interacting systems. Providence, RI: American Mathematical Society. CRM Proc. Lect. Notes. 5, pp. 35-53 (1994)

Burdzy, K., Khoshnevisan, D.: The level sets of iterated Brownian motion. In: Seminaire de Probabilites, XXIX, volume 1613 of Lecture Notes in Math, pp. 231-236. Springer, Berlin (1995)

Curien, N., Konstantopoulos, T.: Iterating Brownian motions, ad libitum. J. Theor. Probab. 27(2), 433-448 (2014)

Dȩbicki, K., Hashorva, E., Ji, L.: Tail Asymptotics of Supremum of Certain Gaussian Processes over Threshold Dependent Random Intervals. Extremes 17(3), 411-429 (2014)

Dębicki, K., Zwart, A.P., Borst, S.C.: The Supremum of a Gaussian Process over a Random Interval. Stat. Prob. Lett. 68, 221-234 (2004) 
Eisenbaum, N., Shi, Z.: Uniform oscillations of the local time of iterated Brownian motion. Bernoulli 5(1), 49-65 (1999)

Funaki, T.: Probabilistic construction of the solution of some higher order parabolic differential equation[s]. Proc. Japan Acad. Ser. A 55, 176-179 (1979)

Hu, Y., Pierre-Loti-Viaud, D., Shi, Z.: Laws of the iterated logarithm for iterated Wiener processes. J. Theor. Probab. 8(2), 303-319 (1995)

Khoshnevisan, D., Lewis, T.M.: Chungs law of the iterated logarithm for iterated Brownian motion. Ann. Inst. H. Poincar Probab. Statist. 32(3), 349-359 (1996)

Khoshnevisan, D., Lewis, T.M.: Iterated Brownian motion and its intrinsic skeletal structure. In: Seminar on Stochastic Analysis, Random Fields and Applications (Ascona, 1996), vol. 45 of Progr. Probab., pp. 201-210. Birkhauser, Basel (1999)

Kozubowski, T.J., Meerschaert, M.M., Molz, F.J., Lu, S.: Fractional Laplace model for hydraulic conductivity. Geophys. Res. Lett. 31, L08501 (2004)

Kozubowski, T.J., Meerschaert, M.M., Podgórski, K.: Fractional Laplace Motion. Adv. Appl. Probab. 38, 451-464 (2006)

Lifshits, M.: Lectures on Gaussian Processes. Springer, Heidelberg (2012)

Linde, W., Shi, Z.: Evaluating the small deviation probabilities for subordinated Lévy processes. Stoch. Process. Appl. 113(2), 273-287 (2004)

Linde, W., Zipfel, P.: Small deviation of subordinated processes over compact sets. Probab. Math. Statist. 28(2), 281-304 (2008)

Michna, Z.: Self-similar processes in collective risk theory. J. Appl. Math. Stoch. Anal. 11, 429-448 (1998)

Nane, E.: Laws of the iterated logarithm for $\alpha$-time Brownian motion. Electron. J. Probab. 11(18), 434-459 (2006). (electronic)

Nourdin, I., Peccati, G.: Weighted power variations of iterated Brownian motion. Electron. J. Probab. 13, 1229-1256 (2008). (electronic)

Pickands, J.: Asymptotic properties of maximum in a stationary Gaussian process. Trans. Amer. Soc. 145, 75-86 (1969)

Piterbarg, V.I.: Asymptotic methods in the theory of Gaussian processes and fields Translations of Mathematical Monographs 148, AMS, Providence (1996)

Shi, Z.: Lower limits of iterated Wiener processes. Statist. Probab. Lett. 23(3), 259-270 (1995)

Tan, Z., Hashorva, E., Peng, Z.: Asymptotics of maxima of strongly dependent Gaussian processes. J. Appl. Probab. 49, 1106-1118 (2012)

Tan, Z., Hashorva, E.: Exact tail asymptotics for the supremum of strongly dependent Gaussian processes over a random interval. Lith. Math. J. 53, 91-102 (2013)

Turban, L.: Iterated random walk. Europhys. Lett. 65(5), 627-632 (2004) 